\title{
SMS Based Remote Monitoring and Control of Industrial Processes using Artificial
}

\author{
Prof Inyiama H.C ${ }^{1}$ and Dimoji D.O \\ ${ }^{1,2}$ Department of Physics/Electronics, Abia State Polytechnic, P. M. B. 7116, Aba, Nigeria. 2School of Industrial \\ Technology, Abia State Polytechnic, P. M. B. 7116, Aba, Nigeria. 3Department of Welding/Fabrication, Abia State \\ Polytechnic, P. M. B. 7116, Aba, Nigeria.
}

\begin{abstract}
This paper presents artificial neural network (ANN) based intelligent control system for effective monitoring and control of industrial processes based on Global System for Mobile Communication network (GSM). This system provides ideal solution for monitoring critical oil/gas plant variables such as temperature, pressure, level and flow- rate. Historical and real time data can be accessed worldwide using the GSM network. The proposed system monitors and controls these variables from the remote location and whenever it crosses the set limit, a microcontroller will sends an SMS to concerned plant personnel(s) mobile phone via GSM network. The concerned personnel can control the system through his mobile phone by sending AT Commands to GSM MODEM and in turn to the microcontroller A Nokia N90 series GSM mobile phone was interfaced with a PC via RS 232 serial port. A PC was used as SMS gateway as well as to create a database to store different records to provide list of changes. An SMS enable was also introduced to enable reporting agent to communicate with the plant. Assembly language, C Language and Visual BASIC were used at different stages to program the necessary hardware. Data obtained from this experiment were analyzed using MATLAB. The performance evaluation and the curve generated with MATLAB shows that using ANN controllers to control industrial Process gives better result/performance than on/off controllers.
\end{abstract}

Keywords: Artificial Neural Network, SMS based monitoring \& control, process control, and remote control.

\section{INTRODUCTION}

On-line monitoring of manufacturing process is extremely important in modern Industrial processes for plant safety, maximization of production and consistency of the product quality Song et al, (2003). There are many processes running in the oil/gas industries due to motor, heater valves etc, we can face serious problems if the devices are not monitored because they can be damaged under abnormal conditions which may result in great loss.
The use of monitoring and control technology in the oil/gas industry has experienced a steady evolution that has paralleled the advances in supervisory control and data acquisition (SCADA) and related technologies during the last 30 plus years. Monitoring and control of remote facilities initially meant a crew (or crews) spending countless hours driving from one remote facility to the next, often on a full-time basis. When they saw a problem at a facility, they fixed it, which often meant making an adjustment to a piece of equipment such as a pump or a valve. The first leap forward was equipping these crews with radios to call in to headquarters when a problem was located, or to call for additional crews when needed. The first steps in automating this process were simple monitoring and alarm systems. These were typically electro- mechanical devices at remote sites that would send a signal back to a control center via radio or leased telephone lines. The GSM has made it possible to send a lot of data from one side of the world to the other side in almost no time.

\section{WHAT IS SMS-BASED MONITORING AND CONTROL?}

The SMS- based monitoring and control (SBMC) can be described as the whole of operations performed to control or monitor a system situated in a closed network.

\subsection{Artificial Neural Networks}

An artificial neural network (ANN) is an information processing paradigm that is inspired by the way biological nervous systems (such as the brain) process information. Christos and Dimitrois (2001) defined ANN as an information processing paradigm that is inspired by the way biological nervous system (such as the brain) process information. It is composed of a large number of highly interconnected processing elements (neurons) working in 
unison to solve specific problems. In biology, the cell body of neuron is called the soma. The spine-like extensions of the cell body are dendrites. The axon extends away from the cell body to provide a pathway for outgoing signals. According to Tonnag (2010) signals are transferred from one neuron to another through a contact point called a synapse. They usually branch repeatedly and from a bushy tree around the body and provide connections to receive incoming signals. ANN are usually called units, node or processing elements, and the efficacy of the synaptic connection, which is a measurement of excitability and inhabitability, are usually called weights. A neural network is composed of a set of nodes connected by weights. The nodes are partitioned into three layers namely input layer, hidden layer and output layer Teo, J. (2005) Teo, J. (2004). Layers are made up of a number of interconnected nodes (artificial neurons or processing element) which contains an activation function.

Figure 1.1 is diagram showing the composition of Artificial Neural Network. ANN is composed of three layers of function. They consist of (a) an input layer, (b) a hidden layer, and (c) an output layer. The hidden layer may consist of several hidden layers as is depicted in Figure 1.1.

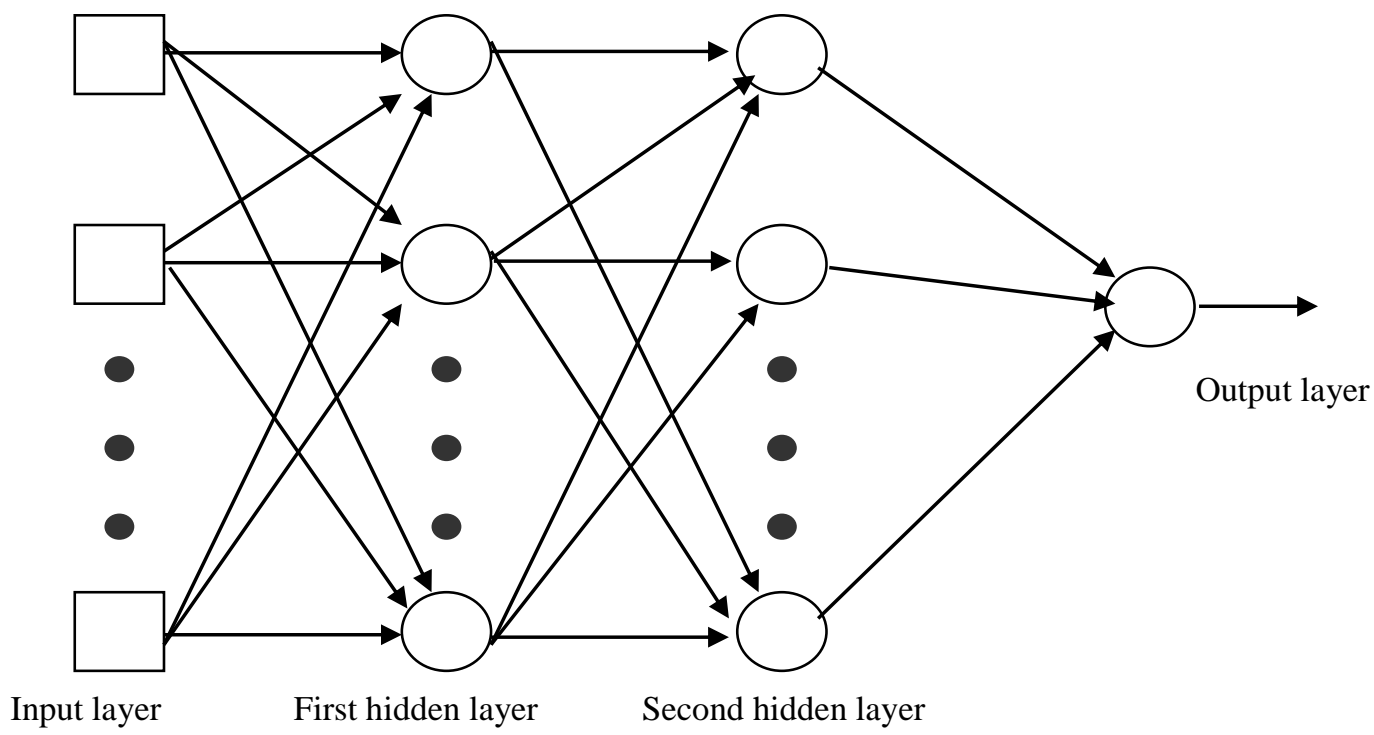

Fig 1.. Two Hidden Layer Multiplayer Perceptron

\subsection{Composition of Artificial Neural Network}

The input layer receives or consists of the input data. It does nothing but buffer the input data. The hidden layers are the internal functions of the ANN. The output layer is the generated results of the hidden layers. The two types of ANN are (a) feed-forward network and (b) a feedback network. The feed-forward ANN has no provision for the use of output from a processing element (hidden layer) to be used as an input for a processing unit in the same or preceding hidden layer. A feedback network allows outputs to be directed back as input to the same or preceding hidden layer. When these inputs create a weight adjustment in the preceding layers, it is called back propagation. An ANN learns by changing the weighting of inputs. During training, the ANN sees the real results and compares them to the ANN outputs. If the difference is great enough, the ANN then uses the feedback to adjust the weights of the inputs. The feedback learning function defines an ANN. The inputs are fed into the input layer and get multiplied by interconnected weights, as they are passed from the input layer to the first hidden layer.

Within the first hidden layer, they get summed then processed by a non-linear function (usually the hyperbolic tangent). As the processed data leaves the first hidden layer, again it gets multiplied by interconnection weights, then summed and processed by the second hidden layer. Finally the data is multiplied by interconnection weights then processed one last time within the output layer to produce the neural network output.

Eliana Zamprogna(2002) used artificial neural networks to describe the behavior of a process. In this research, a soft sensor is developed for a batch distillation column, in order to estimate product compositions using available temperature measurements. A nonlinear deterministic model of the process, which was obtained in a previous study, was used to simulate the dynamic behavior of the batch column under different operating conditions. Kirubashankar etal (2007) proposed a remote monitoring system as part of distributed control system of process 
plant. The following parameter of DCS were monitored continuously: Alarm, graphic display, trend display, system and diagnostic display, control, bar chart, sequence display and fault analysis display. A database is created in MS Access/MY SQL in SCADA for monitoring alarm code, date and time of occurrence of alarm. Taylor eta al (2003) proposed the use of Expert system in Abnormal event management (AEM) in large manufacturing plants. They introduced different computational agents embodied in a three-layered cognitive hierarchy, which offers intelligent behavior at the system level, as well as at the level of specialized task agents. Arshad etal (2001) proposed the application of artificial neural networks in the area of process monitoring, process control and fault detection. This study focused on the malfunctions of the process caused by the failure of the pressure, temperature and cooling water temperature sensors in the reactor. Ramamurthy etal. (2010) proposed a wireless solution, based on GSM (Global System for Mobile Communication) networks for the monitoring and control of humidity in industries.

\section{REQUIREMENT SPECIFICATIONS}

The basic requirements for an industrial automation system are listed as follows:

- It should be able to collect and analyze values of a set of variables. These values are displayed in some way. If the sensor values indicate that some exceptional conditions have arisen then actions are initiated to draw the attention of the operator to that value and certain action(s) to take in response to the exceptional value. Often this involves raising an alarm to draw an operator's attention to the event. Sometimes the system may initiate some other preventive action such as shutting down the system to preserve it from damage.

- It should be able to effectively conduct the desired measurement and control tasks in order to ensure the proper operation of the industrial process.

- It should be able to effectively utilize various signal processing techniques to analyze the gathered data from different measurement points (channels).

- It should be able to increase the software versatility by allowing for flexible configuration of a variety of system parameters.

- It should have comprehensive alarming and reporting capability. The alarm module in the industrial automation software compares the gathered data with the user-set parameters.

\subsection{Functional Block Diagram and Description}

The Functional Block diagram of the entire system is as shown in the Figure 1.2 All the major subsystem blocks are shown with their interconnections to each module .The block diagram consists of temperature, pressure, level and flow-rate Sensors, AT80C51, GSM MODEM (SIM300), MUX, ADC/DAC.SIGNAL CONDITIONING CCT, MAX232 Level converter, Mobile phone, Relay set and Personal computer. In this application, the system was set up to monitor and control the four variables and ensures that they were within safe operating limits. The proposed system consists of several hardware components designed around a central microprocessor. These hardware components include input and output interfaces interfaced with the controller component. The input and output interface links the environment being monitored, the process plant and the reporting agent. The software is made of the artificial network developed using assembly language and $\mathrm{C}$. The control system is centered on the AT89C51 microcontroller. The output interface is made up of LCD and a provision or interfacing to a collating PC via a MAX 232 and GSM module. The sensors gather the values of different parameters like temperature, pressure, level and flow-rate and they are converted into voltage values. Then these parameters are passed to Controller through the DAS. The microcontroller receives the values and sends the corresponding values to the LCD to display. For example if we assign a certain Set-Points for the sensor to monitor the temperature (Ex; Temperature-50 degrees). If the Temperature Sensor crosses its specific Set-Point the microcontroller drives the Fan 'ON' and starts cooling to reach the set point. If the value from the sensor gets below the Set point, the microcontroller drives the heater ' $\mathrm{ON}$ ' and starts the heating to reach the set point. This is a cyclic process. 


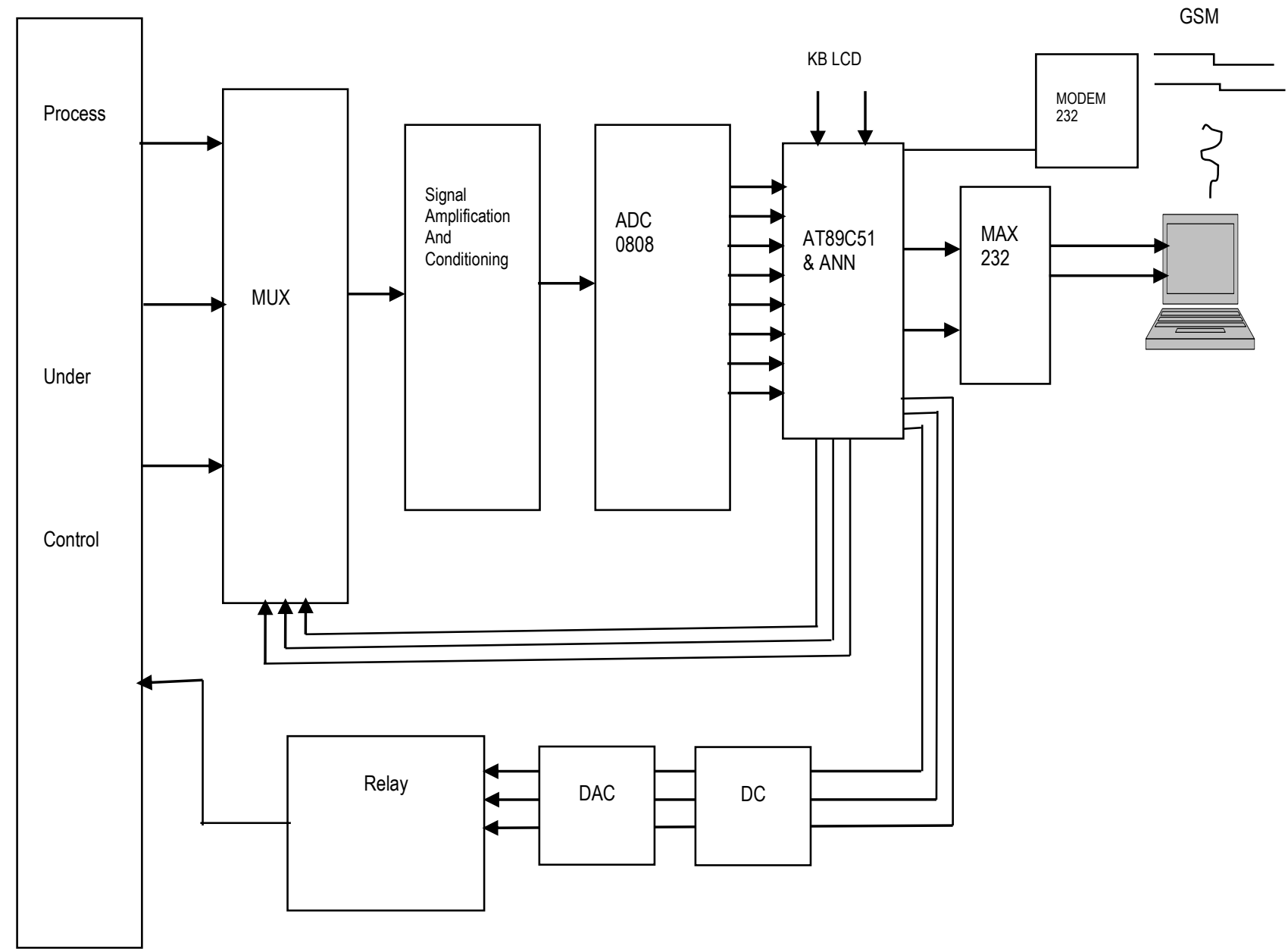

Fig. 2. Block diagram of SMS-Based Remote Process Monitoring and Control Using ANN

\subsection{Design of Control unit Using ANN}

The Control unit consist of ANN based controller. The neural networks classify the input into any one of the seven possibilities. The decision to be taken is in the hidden layer. The ANN is able to classify the range where the neural logic lies. When it is classified, an action corresponding to the class is performed. The control unit interfaces the required values and measured values. The main function of this stage is to keep the actual value close to the required value. The output of this stage is control input for valves and other actuators. In this design, dynamic ANN is used because dynamic ANN is more powerful than static networks because they have memory. They can be trained to learn sequential and time varying factors. The acquired data is initially processed at the data classification module, which uses neural networks and statistics to perform data pre-processing, data compression and data format transfer. It has condition monitoring module, coupled with forward chaining inference, which alerts the user to abnormal process conditions. Then, the incident report module uses the case-based reasoning technique to determine whether the current conditions had previously occurred. If they had, the fault diagnosis module will generate a solution. Otherwise backward chaining will be invoked to detect faults. 


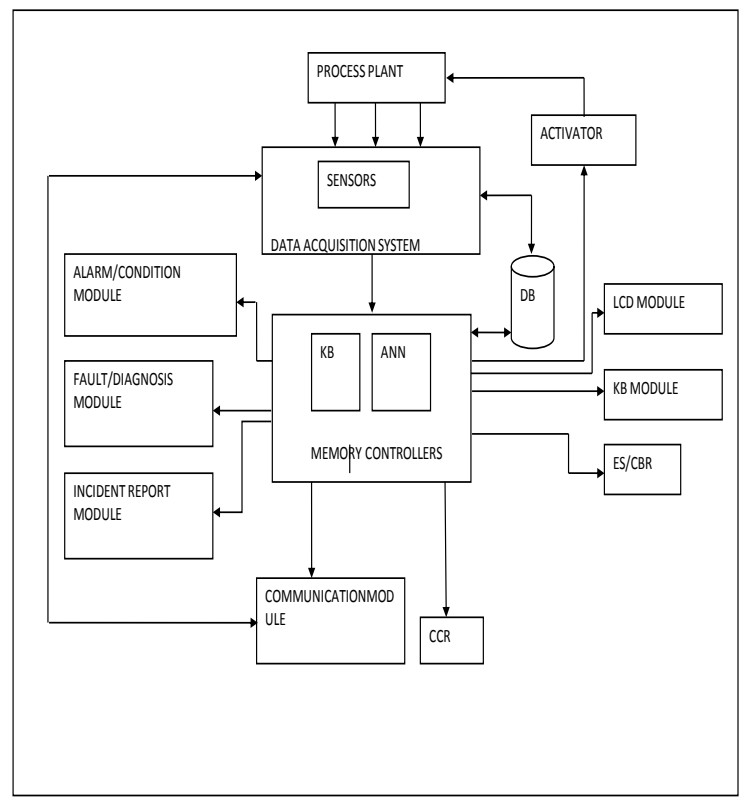

Fig. 3. Block diagram of ANN Based Controller

3.3 Modeling Equation using the back-propagation Algorithm

The activation function of the artificial neurons in ANNs implementing the back propagation algorithm is a weighted sum (the sum of the inputs $x$ multiplied by their respective weights $\mathrm{w}$ )

(1)

$$
A_{j}(\bar{x}, \bar{w})=\sum_{i=0}^{n} x_{i} w_{j i}
$$

We can see that the activation depends only on the inputs and the weights.

Using the common output function, the sigmoidal transfer function: for each node is thus

$$
O_{j}(\bar{x}, \bar{w})=\frac{1}{1+e^{A_{i}(\bar{x}, \bar{w})}}
$$

(2)

The sigmoidal function is very close to one for large positive numbers, 0.5 at zero, and very close to zero for large negative numbers. This allows a smooth transition between the low and high output of the neuron (close to zero or close to one). We can see that the output depends only in the activation, which in turn depends on the values of the inputs and their respective weights.

Now, the goal of the training process is to obtain a desired output when certain inputs are given. Since the error is the difference between the actual and the desired output, the error depends on the weights, and we need to adjust the weights in order to minimize the error. We can define the error function for the output of each neuron:

$$
E_{j}(\bar{x}, \bar{w}, d)=\left(O_{j}(\bar{x}, \bar{w})-d j\right)^{2}
$$

We take the square of the difference between the output and the desired target because it will be always positive, and because it will be greater if the difference is big, and lesser if the difference is small. The error of the network will simply be the sum of the errors of all the neurons in the output layer:

$$
E(\bar{x}, \bar{w}, \bar{d})=\sum_{j}\left(O_{j}(\bar{x}, \bar{w})-d_{j}\right)^{2}
$$

(4)

The back-propagation algorithm now calculates how the error depends on the output, inputs, and weights. After we find this, we can adjust the weights using the method of gradient descendent:

$$
\Delta w_{j i}=-\eta \frac{\partial E}{\partial w_{j i}}
$$

(5)

This formula can be interpreted in the following way: the adjustment of each weight $\mathrm{ji}(\mathrm{w}$ ) will be the negative of a constant eta( $\mathrm{n})$ multiplied by the dependence of their previous weight on the error of the network, which is the derivative of $\mathrm{E}$ in respect to $\mathrm{w}$. The size of the adjustment will depend on $\mathrm{n}$, and on the contribution of the weight to the error of the function. This is, if the weight contributes a lot to the error, the adjustment will be greater than if it contributes in a smaller amount. (5) is used until we find appropriate weights (the error is minimal). So, we "only" need to find the derivative of $\mathrm{E}$ in respect to $\mathrm{w}$. This is the goal of the back-propagation algorithm, since we need to achieve this backwards. First, we need to calculate how much the error depends on the output, which is the derivative of $E$ in respect to $j \& O$ (from (3)).

3.3 Implementation of the procedure for the Artificial Neural Network

The back propagation algorithm was used as a part of implementation in building the artificial neural network for controlling industrial process in oil and Gas process. The neural network program was trained using the back propagation algorithm. This is done by adjusting the weight coefficient until the difference between the desired value and the measured value is within accepted limit. Figure 4.2 is the steps involved in implementing the Artificial Neural Network classifier.. 


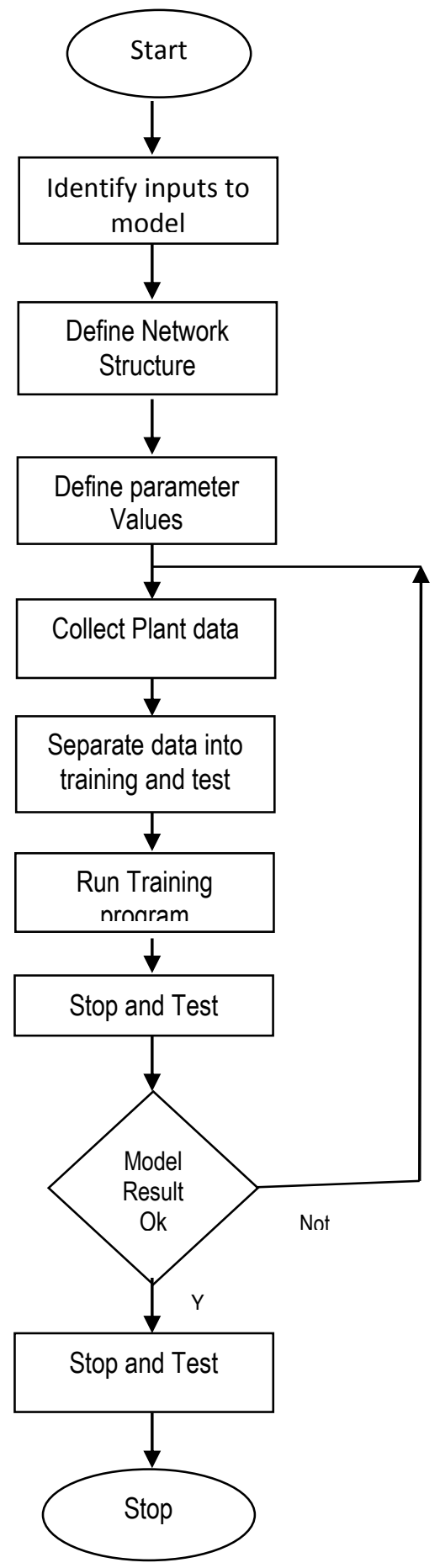

Fig. 4. Flow diagram of the development process of An ANN

\subsection{The Architecture of the Artificial Neural Network}

The architecture for the design of the Work is three inputs, with two hidden layers of seven nodes each, and one output layer making a total of seventeen nodes. This is shown in figure 1.5, three neurons receive inputs to the network. Seven neurons are the weights and seven neurons give outputs from the network. The inputs to the network are Process temperature, pressure level, flow-rate and other manipulated variables. The outputs to the Networks are the-seven possible values of the process variables. There are weights assigned with each arrow which represent information flow. These weights are multiplied by the values which go through each arrow to give more or less strength to the signal which they transmit. The neurons on the output layer receive the outputs of both input neurons, multiplied by their respective weights and sum them.

In other to classify our neurons we chose weight and set them randomly along the network. We change some weights and observe how the behavior of the network changes and which weights are more critical that is if you change those weights, the output will change more dramatically? We provide the network with example of inputs and outputs we want the network to compute the error which is the difference between actual and expected results and is calculated. The idea of the back propagation algorithm is to reduce this error until the ANN learns the training data. The training begins with random weights and the goal is to adjust them so that the error will be minimal. 
The activation function used during training is the sigmoid function defined in equation 1

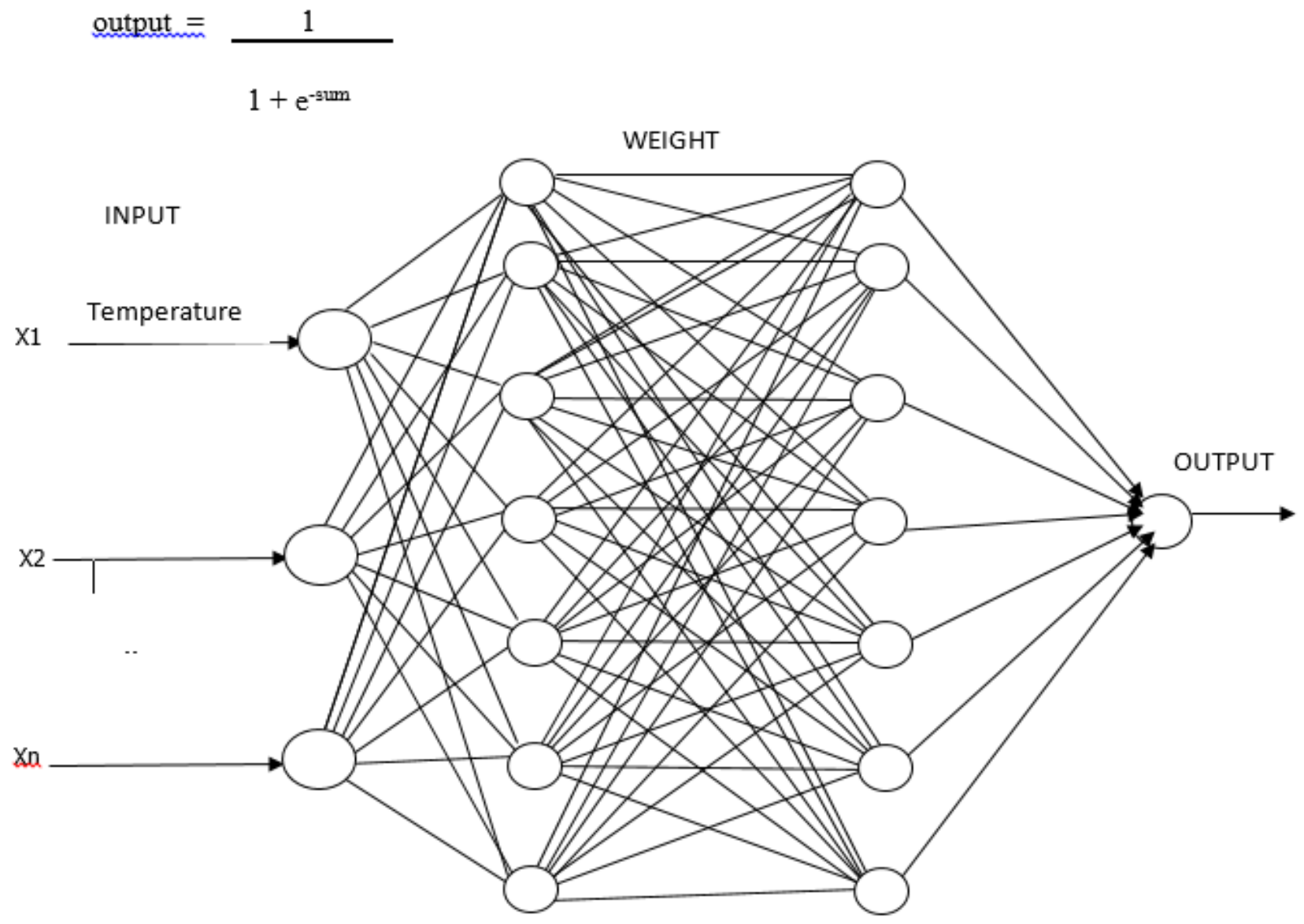

Fig. 5 ANN Classification Diagram for Temperature 


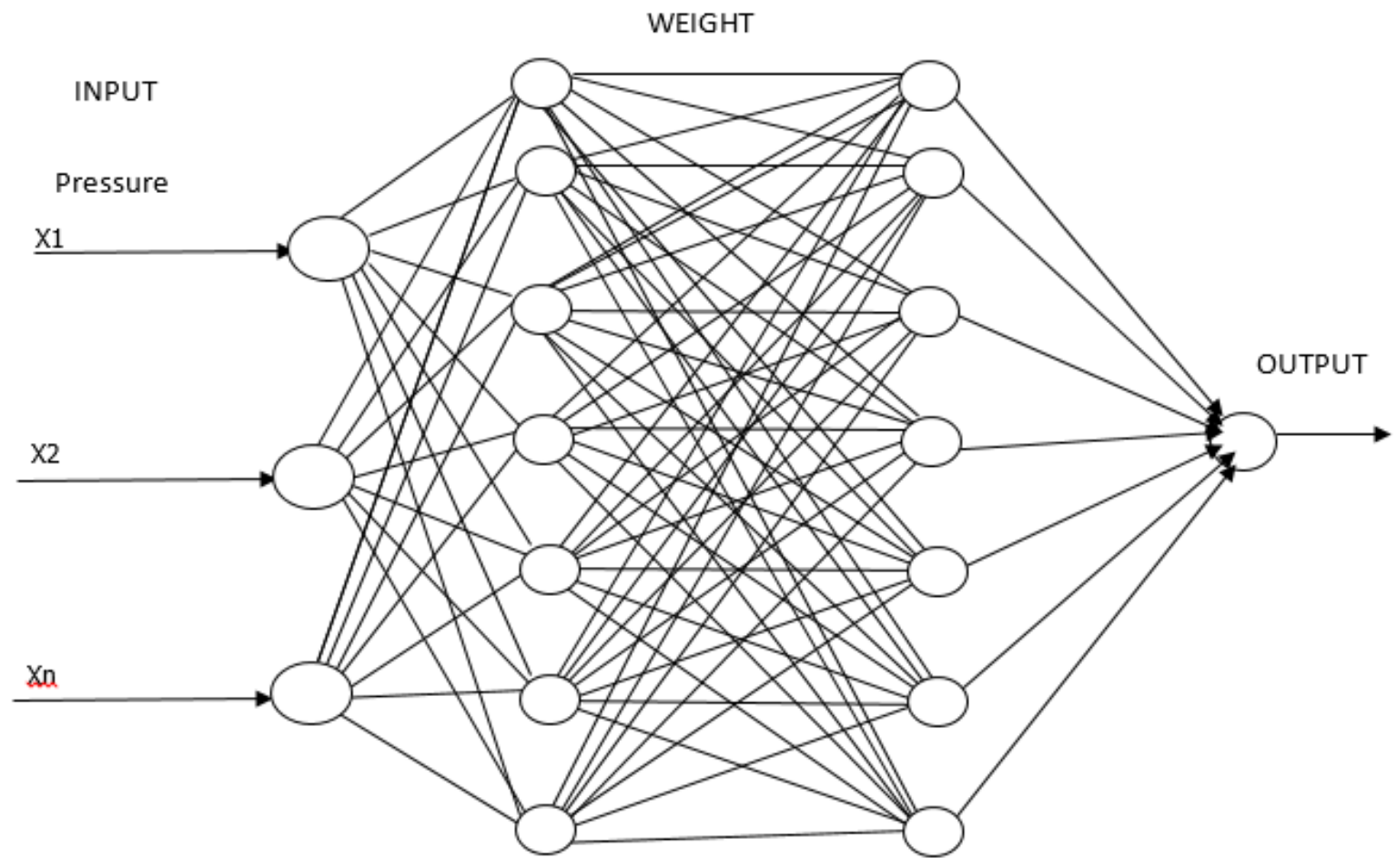

Fig. 6. Neural Network Classification Diagram for Pressure

\subsection{Training of Feedback Neural Network}

In training the ANN for classification a range of values is selected and passed through the network. Any value that does not give correct result is logic error. Then reset the software. A table of the parameter is built each time the software is fine tuned. The neural network is used as classification medium to determine which one should fire. Figure 1.7 shows the firing arrangement. To determine the one that will fire

Send input $\mathrm{X}$, (X can be discrete or continuous
$\mathrm{S}$ passes through output through the activation function $\mathrm{F}(\mathrm{S})$

The activation function can be a step or threshold type that passes through logical 1

If $S>0$ or $S<0$, A positive or negative bias can be introduced to alter threshold value. If the threshold is strong enough, it fires value)

$\mathrm{X}$ passes through synaptic weight $\mathrm{W}$

XW

The network multiplies $\mathrm{X}$ and $\mathrm{W}$ resulting to

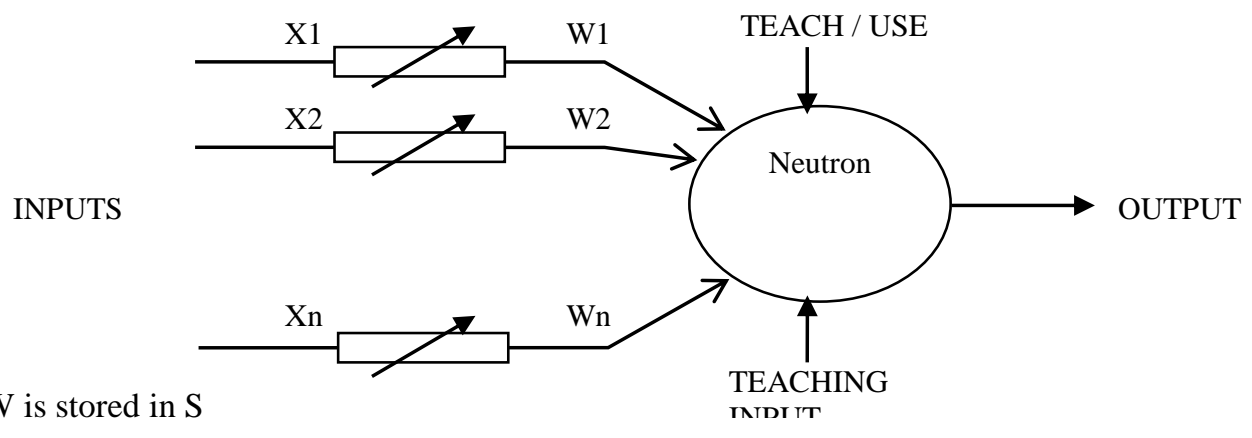

The sum of $\mathrm{XW}$ is stored in $\mathrm{S}$

Fig. 7. Training of Neural Network 


\subsection{Training Data}

The training data for the Artificial Neural Network were collected from the log book of the oil company. The experimental data are the different reading of the plant. Since the nine sub-units are the same and each of them has either two or three variables, we decided to use two sub-units. One with three variables and the other with two variables

Well stream heater (U1)

Temperature

$\begin{array}{lllll}\text { X1 } & \text { X2 } & \text { X3 } & \text { X4 } & \text { SP } \\ 115.0 & 122 & 158 & 166 & 150 \\ 115.5 & 122.5 & 158.5 & 166 & 150 \\ 116.0 & 122.4 & 159 & 166.5 & 150 \\ 116.5 & 122.5 & 159 & 166.5 & 150 \\ 115.0 & 123.0 & 159.5 & 166.5 & 150 \\ 114.0 & 123.0 & 159.5 & 166.5 & 150 \\ 114.0 & 123.5 & 160 & 167 & 150 \\ 114.5 & 122.2 & 160 & 167.5 & 150 \\ 112.0 & 122.6 & 160.5 & 167.8 & 150 \\ 112.0 & 123.5 & 160 . & 168 & 150 \\ 116.0 & 123.5 & 160.7 & 168 & 150 \\ 117.0 & 124 & 160.8 & 168.5 & 150\end{array}$

\section{PRESSURE}

$\mathrm{X} 1$

84.0

84.0

84.0

84.5

84.6

84.8

83.0

83.5

83.5

82.0

82.0

$\begin{array}{llll}\text { X2 } & \text { X3 } & \text { X4 } & \text { SP } \\ 85 & 95 & 96 & 90 \\ 85 & 95 & 96 & 90 \\ 85.5 & 95.2 & 96.2 & 90 \\ 85.8 & 95.2 & 96.2 & 90 \\ 88.0 & 95.5 & 96.4 & 90 \\ 86.0 & 95.5 & 95.4 & 90 \\ 86.4 & 95.6 & 96.8 & 90 \\ 86.6 & 95.6 & 97.0 & 90 \\ 86.8 & 95.8 & 97.0 & 90 \\ 86.8 & 95.8 & 97.5 & 90 \\ 87.0 & 95.8 & 97.5 & 90\end{array}$

\subsection{The Control Flow Chart}

Figure 1.8 is the block diagram form how the program for the microcontroller with three loops is structured to service each of the cases or loops. For each of the case/loop, you select the variable, condition the analogue signal value; convert the signal to its digital value using ADC. The processor reads the value, then determines the $\mathrm{K}$ value and performs action corresponding to $\mathrm{K}$ value. The microcontroller services each loop at regular intervals. Each loop is controlled by a different procedure.

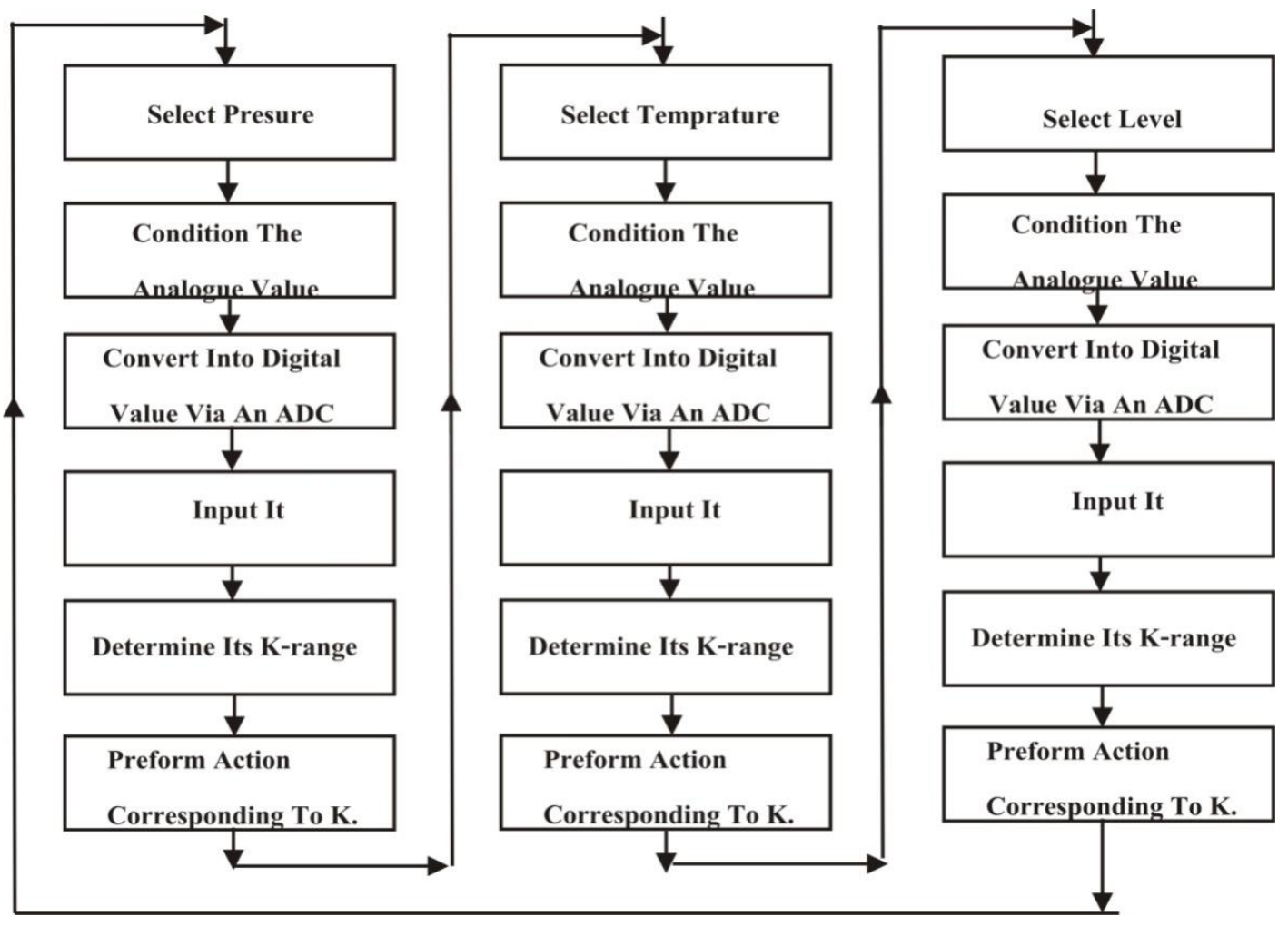

Fig. 8. Block Diagram Of ASM Control Chart. 


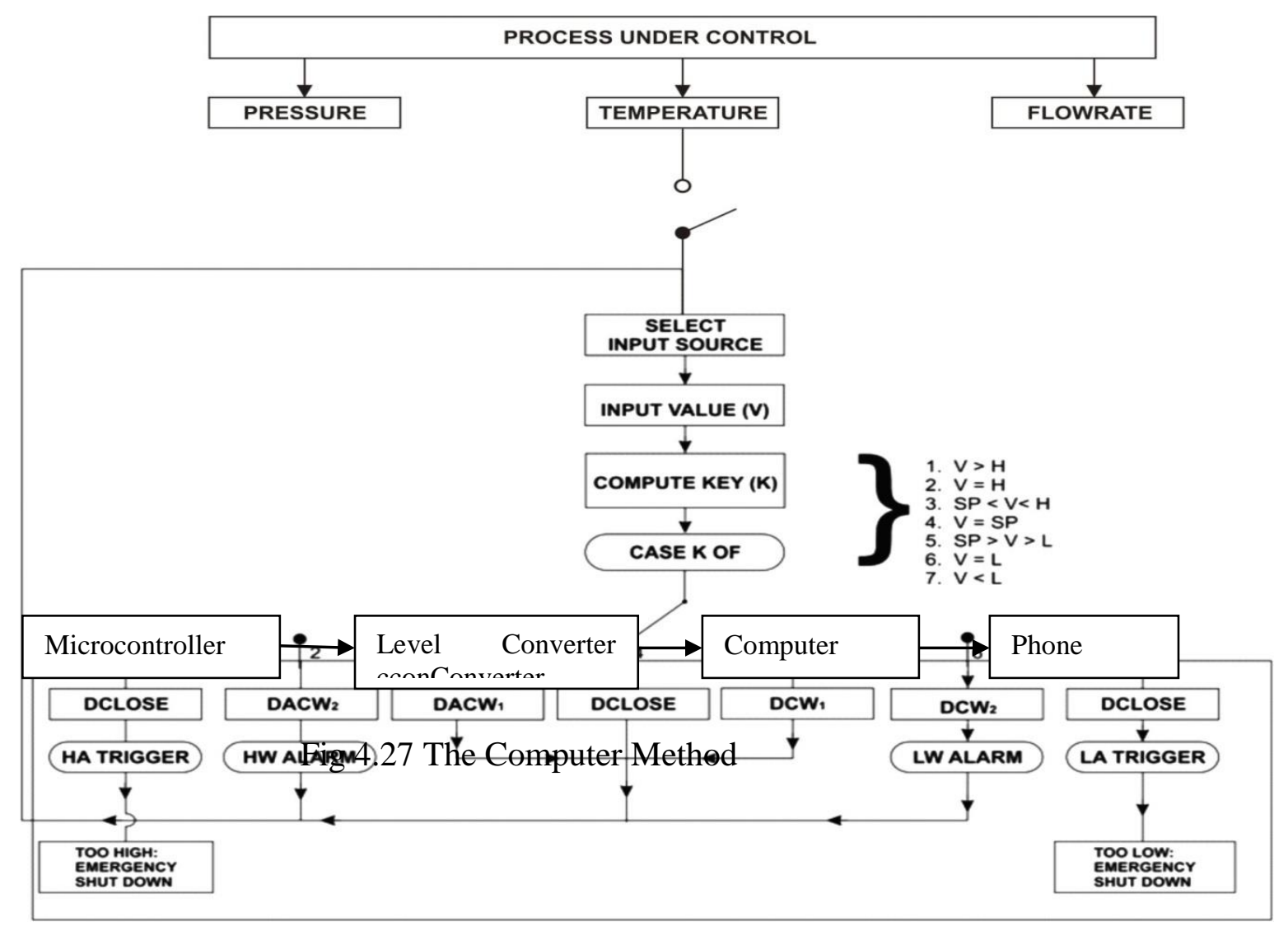

Fig. 9. ASM Chart for the Process

\subsection{The process control Algorithm}

The ASM control chart is the procedure that the processor uses to monitor and control multiple process variables. An input source is selected which may be, Temperature, Pressure, or Level value and is read by the processor and the key is computed.

They are seven keys as indicated in ASM control chart namely:
1. Value $(\mathrm{V})>\operatorname{High}(\mathrm{H})$
2. Value $(\mathrm{V})=$ High.
3. $\mathrm{SP}<$ Value $<\mathrm{H}$
4. $\mathrm{V}=\mathrm{SP}$
5. $\mathrm{SP}>\mathrm{V}>\mathrm{L}$
6. $\mathrm{V}=\mathrm{L}$
7. $\mathrm{V}<\mathrm{L}$

The case that will be selected will depend on the key.

If $\mathrm{V}>\mathrm{H}$, case 1 is selected, and the device will be closed and the high Alarm device will be triggered and there will be Emergency shutdown.

If Value $=$ High, case 2 is selected, the Device will be set Anti clockwise twice low warning Alarm

If $\mathrm{SP}<$ Value $<\mathrm{H}$ case 3 is selected and device is set anti clockwise once.

If $\mathrm{V}=\mathrm{SP}$, case 4 is selected, the Device is close.
If $\mathrm{SP}>\mathrm{V}>\mathrm{L}$, case 5 is selected, the Device is turned Click me once and returned back to selected input source. If $\mathrm{V}=\mathrm{L}$, case 6 is selected, that is value is equal to low. The device is turned Clockwise twice; a low alarm working is sounded

If $\mathrm{V}<\mathrm{L}$ case 7 is selected $(\mathrm{V}>\mathrm{L})$ the DLA Trigger is sounded and there will be an emergency short down of the plant.

For any of the cases selected, the block diagram in figure $4.20 \mathrm{~b}$ will be executed. Each of the case is a loop..

\section{THE PC INTERFACE}

The PC serves as SMS gateway and also hosts a database to store various diagnostic information and archival data for decision making. The computer method was adopted in this work. This method involves the use of level converter to interface between the microcontroller and the computer. The computer has the application program that gets data from the controller and in turn send appropriate SMS message using Attention Command (AT) via the phone. Figure 4.27 is the Computer Method. 


\subsection{SMS Interface}

The widely available networks are based on GSM. The network provides a wide area of coverage and can be utilized more cost-effectively for this system and communication protocols that are DTMF (Dual Tone Multi Frequencies) (R. Sharma, K. Kumar, et al 2006), In this work the MTN network was used because of it wide coverage.

\subsection{Design of SMS Interface}

The GSM subsystem is interfaced with the Microcontroller using a RS232 interface. Since the RS232 is not compatible with today's Microprocessors and Microcontrollers, we need a voltage converter to convert RS232"es signals to TTL voltage levels. MAX 232 was used as a voltage converter. The MAX232 converter converts from RS232 voltage levels to TTL voltage levels and vice versa. One advantage of the MAX232 chip is that it uses a +5 v power source, which is the same as the source voltage for the microcontroller. In other words, with a single $+5 \mathrm{v}$ power supply we can power both the microcontroller and MAX232, with no need for the dual power supplies that are common in many older systems. The MAX 232 has two sets of line drivers for transferring and receiving data. The GSM has a SIM (Subscriber Identity Module) card to make the network identify the user. A Nokia N90 series GSM modem was also interfaced to the PC to facilitate interaction with system/maintenance engineers anywhere they maybe, anytime it becomes necessary. The microcontroller communicates with the GSM module using the AT commands and other high level language,. These AT commands are used to send and receive SMS. The programming code for the microcontroller is written in some high level language. The GSM module has an RS232 interface for serial communication with an external peripheral. In this case, the transmit pin (Tx) of the computer's Serial port is connected with the receive pin ( $\mathrm{Rx})$ of the GSM module's RS-232 interface. The transmit pin (Tx) of the RS-232 of GSM module is connected to receive pin (Rx) of microcontroller's serial transmission pin. And the serial transmit pin of the microcontroller is connected to the receive pin of the computer's Serial port.

\subsection{System Architecture for SBMC System}

In using the GSM for remote process control system using SMS, the remote personnel receives the status report of the process through SMS message from the user Mobile Phone, The user sends a command to controller to control the process variable by sending a set command. When action is taken, a feedback report is sent to the user. In a situation where the user cannot effect control, the attention of the control room can be drawn through the same means.

Figures $1.9 \mathrm{a}, 1.9 \mathrm{~b}$ and $1.9 \mathrm{c}$ illustrates the temperature, pressure and level control of the stream well of the system from the data in table 5.1using MATLAB 7.1.

\subsection{Temperature Control}

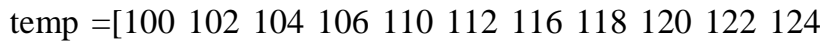

126135140145148150151152154148149150152

$154150155160158156]$;

$>>$ time $=[0: 2: 58]$;

plot(time,temp)

xlabel('Time(sec)')

ylabel('Temp(oC)')

grid on

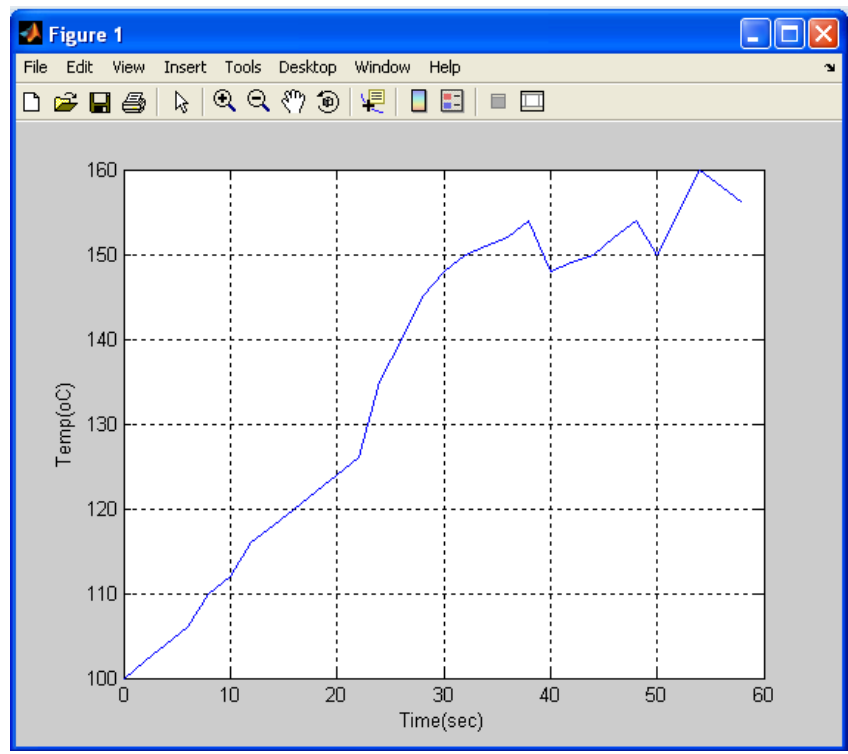

Fig. 4a. Temperature Control curve of set point of 150

The curve in fig 5.5a shows the temperature of a process in gas tank. The temperature was taken after five seconds interval .The set point is $150{ }^{\circ} \mathrm{C}$ with $4 \%$ either way. The curve shows a negligible offshoot.

\subsection{Level Control}

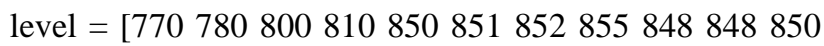
850855860 900];

time $=[0: 14]$

plot (time, level)

xlabel ('Time(sec)')

ylabel ('Level (m)')grid on 


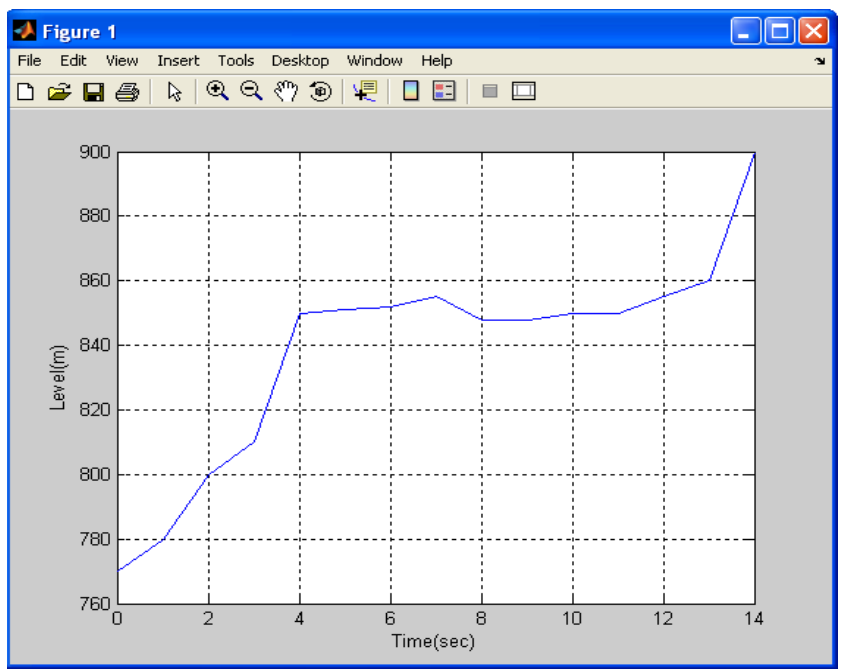

Fig. 5b. Level Control Curve with set point of 850

The Figure 5.5b shows a Level Control curve of set point of 850. The curve generated by the microcontroller demonstrated better result in terms of stability.

\subsection{Pressure Control}

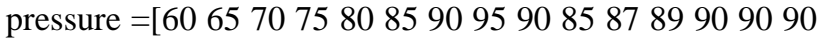
90 90];

plot(time, pressure)

$>$ time $=[5: 5: 85]$

>> xlabel ('Time(sec)')

$>$ ylabel('Psi')

$>$ ylabel('Pressure(psi)')

> grid on

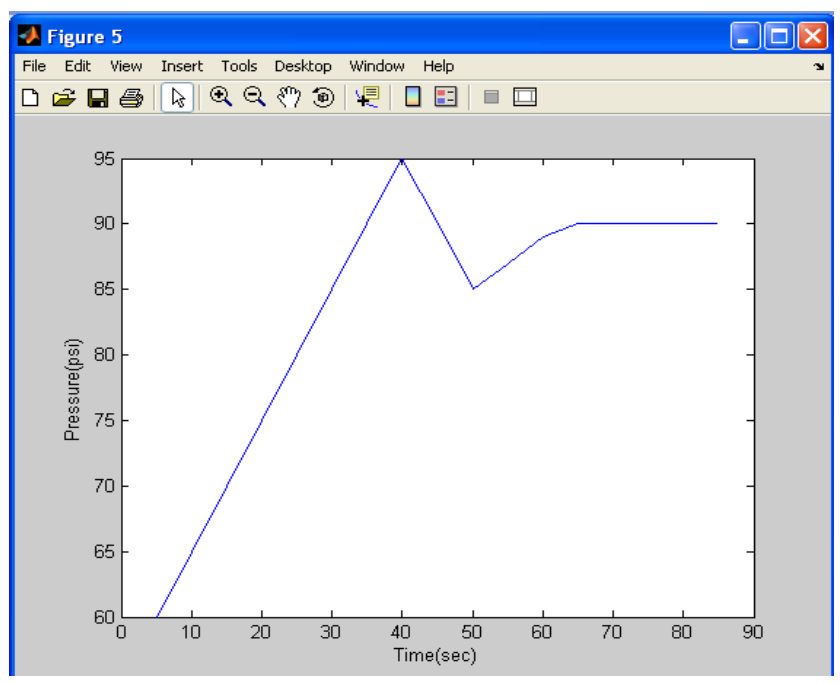

Fig. 4c. Graph of pressure Control curve with a set point of 90

The curve in $5.5 \mathrm{c}$ above shows the control operation of the pressure variable in the stream well with a set point of
90 psi. The curve demonstrated that the overshoot is within limits.

\section{CONCLUSION}

This paper has described an approach to remote industrial process monitoring and control using Artificial Neural Networks. It has explored and demonstrated the utility of artificial neural networks technology for developing effective controller for dynamic industrial processes. The work demonstrated that it is possible to design a system that will monitor industrial variables such as pressure, temperature, level and flow rate in an Oil and Gas plant and alert remote personnel (in case of departure from set points) who can possibly make a change. As result of the use of multi-processors it was possible to keep each of the monitored variables within set limits using Artificial Neural Network approach. The design of an SMS-Based software to alert industrial personnel when and if any of these variables goes out of bounds in spite of the control systems effort has been shown. The system is self learning; self adaptive and self diagnosing using Rule based expert system. The new system is compared with ON/OFF controller and it is shown that ON/OFF Controller based system fails miserably because of its limitations. On the other hand ANN based approach has resulted in possible implementation of better and more efficient control.

\section{REFERENCES}

[1] Song, S. O.; Lee, G. \& Yoon, E.N. (2004). Process Monitoring of an Electro-Pneumatic Valve Actuator Using Kernel Principal Component Analysis, Proceedings of the International Symposium on Advanced Control of Chemical Processes. Hong Kong,China, 11-14 January 2004.

[2] S. Christos, S. Dimitrios, Introduction to Artificial Neural Network,, Research Report (2001)

[3] Z.E.H. Tonnang Distillation Column Control Using Artificial Neural Networks, M. Sc Thesis, Microprocessors and Control Engineering, Department of Electrical and Electronics Engineering, Faculty of Technology, University of Ibadan, Ibadan, Nigeria (2010).

[4] Teo, J. Evolutionary Multi-Objective Optimization for Automatic Synthesis of Artificial Neural Network Robot Controllers. Malaysian Journal of Computer Science, volume 18, number 2, 2005, pp 54-62.

[5] Teo, J. -Darwin + Robots = Evolutionary Robotics: Challenges in Automatic Robot Synthesisl. 2nd International Conference on Artificial Intelligence in Engineering and Technology (ICAET 2004). Kota 
Kinabablu, Sabah, Malaysia, August 2004, Vol 1, pp 713.

[6] Kirubashankar, R Krishnemunthy K and India J (2009) "Remote Monitoring system for distributed Control of Industrial Plant Process" Journal of Scientific and Industrial Research Vol 68, PP -SS6- 860.

[7] Dr.B.Ramamurthy1 S.Bhargavi2 Dr.R.ShashiKumar 3

Dept of Instrumentation, S K University Dept of E\&C, SJCIT Dept of E\&C, SJCIT

[8] Anantapur, Andhra Pradesh India Chikballapur, Karnataka, India Chikballapur, K

International Journal of Advanced Computer Science and Applications,

Vol. 1, No. 4, October 2010

[9] B.A. Zalizawati, Development of Multiple-Input Multiple-Output and Multiple-Input Single-Output Neural Network Models for Continuous Distillation Column, M. Sc. Thesis, School of Chemical Engineering, Malaysia University, Malaysia (2008).

[10] S.Muhammad Umair R. Usman Automation of Irrigation System Using ANN based Controller. Research Centre for Modeling \& Simulation, National University of Science and Technology, Pakistan International Journal of Electrical \& Computer Sciences IJECS-IJENS Vol:10 No:02 45(2010)

[11] James H. Taylor1 and Atalla F. Sayda2 (2006) Intelligent Information, Monitoring, and Control Technology of Industrial Process Applications.Department of Electrical \& Computer Engineering University of New Brunswick PO Box: 4400, Rm. H113 Head Hall Fredericton, NB CANADA E3B 5A3

[12] Harish Ramamurthy, B. S. Prabhu and Rajit Gadhv Asad M. MadniB(2007) Wireless Industrial Monitoring and Control using a Smart Sensor Platform. Wireless Internet for the Mobile Enterprise Consortium University of California, Los Angeles USA. EI Technologies, Inc.Sylmar, California, USA

[13] Arshad Ahmad a, Mohd Kamaruddin Abd Hamid(2001) Neural Networks for Process Monitoring, Control and Fault Detection: Application to Tennessee Eastman Plant. Center for Process Simulation, Department of Chemical Engineering, Faculty of Chemical \& Natural Resources Engineering, Universiti Teknologi Malaysia, 80990 Malaysian Science and Technology Congress, Melaka, 2001. 